José Gilberto Pereira ${ }^{1}$

Vera Lúcia Edais Pepe ${ }^{1}$

\title{
ACESSO A MEDICAMENTOS POR VIA JUDICIAL NO PARANÁ: APLICAÇÃO DE UM MODELO METODOLÓGICO PARA ANÁLISE E MONITORAMENTO DAS DEMANDAS JUDICIAIS
}

Judicial access to medicine in Paraná: applying a methodological model for lawsuit analysis and monitoring

${ }^{1}$ Escola Nacional de Saúde Pública, Fundação Oswaldo Cruz. Rio de Janeiro/RJ, Brasil. Correspondência: José Gilberto Pereira. E-mail: jose.gilberto.pereira@gmail.com.

Recebido em: 03/06/2013. Aprovado em: 17/12/2013. 


\section{RESUMO}

$\mathrm{O}$ número crescente de demandas judiciais individuais por medicamentos tem exigido estudos de caracterização e análise. As diferentes abordagens e análises realizadas dificultam a comparação entre estados e/ou municípios brasileiros, fato este que inspirou a construção de indicadores de análise e monitoramento dessas demandas. O objetivo deste trabalho foi aplicar tal modelo metodológico na análise das demandas judiciais individuais para o acesso a medicamentos no Estado do Paraná. Para tanto, foi realizado um estudo empírico de análise das demandas judiciais por medicamentos junto à Secretaria de Estado da Saúde referentes ao ano de 2009. A partir da identificação e quantificação de variáveis selecionadas, foram calculados os indicadores propostos para avaliação e monitoramento das demandas judiciais de medicamentos. Procedeu-se, então, à análise da qualidade do modelo desenvolvido, com base na variabilidade das fontes de informação, na completude dos dados e na seleção da unidade de análise. Devido à indisponibilidade de informações nas fontes selecionadas, não foi possível determinar todos os indicadores propostos - em particular os relacionados às características sociodemográficas dos autores das ações. Para o uso eficiente do conjunto de indicadores, como instrumento tanto de pesquisa quanto de gestão, seria necessário dispor da cópia de cada processo judicial na íntegra.

\section{Palavras-chave}

Assistência Farmacêutica; Decisões Judiciais; Direito à Saúde; Indicadores de Avaliação e Monitoramento; Sistema Único de Saúde.

\section{ABSTRACT}

The growing number of individual lawsuits over medicines has required characterization and analysis studies. The different approaches and analyses hinder the comparison between Brazilian states and/or municipalities, thus inspiring the elaboration of indicators to analyze and monitor these claims. This study aims at applying the referred methodological model to individual lawsuit analysis on medicine access in Paraná. Accordingly, we conducted an empirical analysis on the medicine access lawsuits addressed to the Health Secretariat of State, relative to 2009. After the identification and quantification of selected variables, we calculated the proposed indicators to evaluate and monitor the medicine lawsuits. In addition, the developed model quality was analyzed based on information source variability, data completeness, and the selection of the analysis unit. Due to information unavailability from the selected sources, it was not possible to determine all the proposed indicators-in particular, those related to the social and demographic characteristics of the concerned plaintiffs. To effectively use the set of indicators, both as research and management instrument, it would be necessary to have a complete available copy of each lawsuit.

\section{Keywords}

Pharmaceutical Service; Court Decisions; Right to Healthcare; Monitoring and Evaluation Indicators; Unified Healthcare System 


\section{Introdução}

A crescente demanda por serviços e insumos de saúde nas últimas décadas no Brasil inclui os medicamentos, para diversos tipos de indicações terapêuticas ${ }^{1,2}$. O debate jurídico travado no exame dos pedidos judiciais de medicamentos, em conjunto com as ações administrativas de enfrentamento da questão, têm-se tornado, de maneira crescente, objeto de investigação acadêmica e científica. Estudos sobre a demanda judicial contra as três esferas de governo têm abordado desde a caracterização mais geral das demandas em estados e municípios ${ }^{3,4,5}$ até análises mais específicas, como a demanda por medicamentos do componente especializado, classes específicas de medicamentos e medicamentos sem registro ${ }^{6,7}$.

Já foi possível identificar características comuns, tais como o crescente número de demandas judiciais e/ou administrativas e o progressivo gasto da União, dos estados e dos municípios com elas. Assim é que tanto na esfera federal como nos principais estados, como São Paulo e Rio de Janeiro, os gastos com medicamentos demandados por meio da Justiça têm sido da ordem de milhões, acompanhando o crescimento de demandas judiciais e/ou administrativas ${ }^{8,9,10}$.

Também foram identificadas diferenças, por exemplo, relativas aos representantes legais dos autores da ação, à prescrição de serviço público ou privado e às características socioeconômicas dos autores. Além disso, as variadas formas de apresentação dos resultados dos estudos realizados dificultam compor um quadro

${ }^{1}$ ANDRADE, Eli lola Gurgel et al. A judicialização da saúde e a política nacional de assistência farmacêutica no Brasil: gestão da clínica e medicalização da justiça. Revista Médica de Minas Gerais, Belo Horizonte, v. 18, supl. 4, p. 46-50, 2008.

${ }^{2}$ FERRAZ, Octávio Luiz Motta; VIEIRA, Fabíola Sulpino. Direito à saúde, recursos escassos e equidade: os riscos da interpretação judicial dominante. Dados, v. 52, n. 1, p. 223-251, mar. 2009.

${ }^{3}$ LEITE, Silvana Nair et al. Ações judiciais e demandas administrativas na garantia do direito de acesso a medicamentos em Florianópolis-SC. Revista de Direito Sanitário, São Paulo, v. 10, n. 2, p. 13-28, 2009.

${ }^{4}$ FIGUEIREDO, Tatiana Aragão. Análise dos medicamentos fornecidos por mandado judicial na Comarca do Rio de Janeiro: a aplicação de evidências científicas no processo de tomada de decisão. 2010. Dissertação (Mestrado) - Escola Nacional de Saúde Pública Sérgio Arouca, Fundação Oswaldo Cruz. Rio de Janeiro, 2010.

${ }^{5}$ MACHADO, Marina Amaral de Ávila et al. Judicialização do acesso a medicamentos no Estado de Minas Gerais, Brasil. Revista de Saúde Pública, São Paulo, v. 45, n. 3, p. 590-598, 2011.

${ }^{6}$ CAMARGO, lara Alves. Análise das demandas judiciais para o tratamento da artrite reumatoide no Estado de São Paulo. 2011. Dissertação (Mestrado) - Universidade de Sorocaba. Sorocaba, 2011.

${ }^{7}$ ANJOS, Renata Sacco dos. Judicialização e equidade no tratamento da hepatite C: estudo de caso sobre o tratamento com interferona alfa em um serviço de referência do SUS em Porto Alegre, RS, 2009. Dissertação (Mestrado) - Faculdade de Medicina, Universidade Federal do Rio Grande do Sul. Porto Alegre, 2009. ${ }^{8}$ ADVOCACIA GERAL DA UNIÃO - AGU. Intervenção judicial na saúde pública: panorama no âmbito da Justiça Federal e apontamentos na seara das Justiças Estaduais, 2012. Disponível em: <http://portalsaude. saude.gov.br/portalsaude/arquivos/pdf/2013/Abr/26/Panoramadajudicializacao2013.pdf>. Acesso em: 20 maio 2013.

${ }^{9} \mathrm{CHIEFFI} \mathrm{Ana} \mathrm{Luiza;} \mathrm{BARATA} \mathrm{Rita} \mathrm{de} \mathrm{Cássia} \mathrm{Barradas.} \mathrm{Ações} \mathrm{judiciais:} \mathrm{estratégia} \mathrm{da} \mathrm{indústria} \mathrm{farma-}$ cêutica para introdução de novos medicamentos. Revista de Saúde Pública, São Paulo, v. 44, n. 3, p. 421-429, 2010.

${ }^{10}$ TEIXEIRA, Mariana Faria. Criando alternativas ao processo de judicialização da saúde: o sistema de pedido administrativo, uma iniciativa pioneira do Estado e Município do Rio de Janeiro. 2011. Dissertação (Mestrado) - Escola Nacional de Saúde Pública Sérgio Arouca, Fundação Oswaldo Cruz. Rio de Janeiro, 2011. 
nacional e mesmo aprofundar a análise das semelhanças e diferenças entre as distintas regiões estudadas ${ }^{11}$.

Visando permitir análises mais homogêneas de algumas características importantes das demandas judiciais de medicamentos, foi desenvolvida e validada, em consenso de especialistas, uma proposta de 30 indicadores básicos, distribuídos em quatro dimensões - sociodemográficas, processuais, médico-sanitárias e político-administrativas - das ações judiciais para avaliação e monitoramento das demandas de medicamentos. Uma das etapas desse processo é a identificação e a padronização de variáveis, o que resultou na elaboração do Manual de Indicadores de Avaliação e Monitoramento das Demandas Judiciais de Medicamentos ${ }^{12}$. O presente artigo tem como objetivo aplicar o modelo metodológico desenvolvido na análise das demandas judiciais individuais para o acesso a medicamentos no Estado do Paraná.

\section{Métodos}

O estudo empírico foi do tipo descritivo transversal de análise das demandas judiciais por medicamentos junto a Secretaria de Estado da Saúde do Paraná (SESA/PR) referentes ao ano de 2009.

As fontes primárias de informação foram os bancos de dados dos mandados judiciais do Centro de Medicamentos do Paraná (Cemepar), e as fontes secundárias complementares foram os dados processuais disponíveis nos sítios da Associação dos Serventuários da Justiça do Estado do Paraná (Assejepar) ${ }^{13}$, do Tribunal de Justiça do Estado do Paraná (TJ-PR) ${ }^{14}$ e do Tribunal Regional Federal da $4^{\mathrm{a}}$ Região (TRF-4) ${ }^{15}$ na internet. A unidade de análise foi demanda judicial individual, identificada pelo nome do demandante ou pelo número do processo judicial. O aplicativo computacional do Cemepar, que registra e gerencia a demanda judicial, também abrangeu 174 demandas administrativas (extrajudiciais) utilizadas no cálculo de um indicador.

Dados de custos financeiros para aquisição dos medicamentos foram obtidos no sítio do Tribunal de Contas do Estado do Paraná (TCE-PR) ${ }^{16}$ na internet. A coleta

\footnotetext{
${ }^{11}$ SANT'ANA, João Maurício Brambati. Essencialidade e assistência farmacêutica: um estudo exploratório das demandas judiciais individuais para acesso a medicamentos no Estado do Rio de Janeiro. Dissertação (Mestrado). Escola Nacional de Saúde Pública Sergio Arouca, Fundação Oswaldo Cruz. Rio de Janeiro, 2009.

${ }^{12}$ PEPE, Vera Lúcia Edais; VENTURA, M. (Org.). Manual indicadores de avaliação e monitoramento das demandas judiciais de medicamentos. Rio de Janeiro: Fundação Oswaldo Cruz, Escola Nacional de Saúde Pública Sergio Arouca, 2011.

${ }^{13}$ ASSOCIAÇÃO DOS SERVENTUÁRIOS DA JUSTIÇA DO ESTADO DO PARANÁ - ASSEJEPAR. Consulta Processual. Disponivel em: <http://www.assejepar.com.br>. Acesso em: 20 ago. 2011.

${ }^{14}$ PARANÁ. Tribunal de Justiça do Estado. Consulta Processual. Disponível em: <http://www.tjpr.jus.br/consulta-processual>. Acesso em: 20 ago. 2011.

${ }^{15}$ TRIBUNAL REGIONAL FEDERAL DA 4 ${ }^{a}$ REGIÃO. Consulta Processual. Disponível em: <http://www2.trf4.jus. br/trf4/>. Acesso em: 20 ago. 2011.

${ }^{16}$ PARANÁ. Tribunal de Contas do Estado. Contas do Governador: relatório e parecer prévio, Exercício 2009. Curitiba: Tribunal de Contas do Estado; Instituto Rui Barbosa. 2010. p. 179. Disponível em: <http://www. tce.pr.gov.br/contasdogoverno2009>. Acesso em: 16 out. 2012.
} 
de dados foi orientada pela lista de variáveis utilizadas para o cálculo de todos os indicadores descritos no Manual já citado. Os dados foram coletados, registrados e analisados no período de 20 de agosto de 2011 a 20 de novembro de 2012.

Os arquivos do Cemepar continham informações selecionadas com base em um formulário próprio denominado "ficha técnica para cumprimento de ordem judicial", que trazia as seguintes variáveis: domicílio (característica sociodemográfica); decisão liminar/antecipação de tutela, natureza da ação judicial, natureza do representante judicial do autor da ação, réu(s) da ação, tempo de tramitação da ação judicial, tempo de decisão liminar, tempo da intimação da instância da saúde, número de ações por ano de competência, condutor da ação (características processuais); classificação da doença segundo a CID-10, classificação dos medicamentos segundo a Anatomical Therapeutic Chemical (ATC), número de medicamentos prescritos pelo nome genérico ou pela relação de Denominações Comuns Brasileiras (DCB) (35,2\% dos arquivos individuais não possuíam cópia da prescrição), número de medicamentos prescritos presentes na Relação Nacional de Medicamentos Essenciais (Rename) vigente, medicamentos prescritos presentes nas listas estaduais ou municipais de medicamentos essenciais vigentes, racionalidade da indicação terapêutica (características médico-sanitárias); medicamentos registrados na Agência Nacional de Vigilância Sanitária (Anvisa), origem da prescrição médica segundo o sistema de saúde (limitada à presença da cópia da prescrição), número de medicamentos por componente do bloco de financiamento da assistência farmacêutica, existência de alternativa terapêutica ao medicamento requerido entre os medicamentos padronizados (características político-administrativas).

Além das variáveis necessárias ao cálculo dos indicadores, também foram identificadas e quantificadas nas fontes de informação variáveis de interesse ao campo do Direito: comarca; tipo de vara (Cível, Criminal, Família, Infância e Juventude, Fazenda Pública e Federal); tipo de ação impetrada (civil pública, cominatória, de conhecimento, de obrigação de fazer, mandado de segurança, ordinária e outros tipos); justiça gratuita; aplicação de medida constritiva; apelação da sentença ao tribunal do segundo grau; e número de réus na ação.

As seguintes variáveis foram coletadas nos bancos de dados processuais eletrônicos da Assejepar, do TJ-PR e do TRF-4: natureza da autoria da ação, sentença, acórdão, gratuidade de justiça (características processuais); outros documentos médicos apensados ao processo (características médico-sanitárias). Já a variável "gastos anuais para aquisição dos medicamentos requeridos" (característica político-administrativa) foi obtida no relatório anual do TCE-PR.

Os indicadores determinados, as variáveis identificadas e sua aplicação foram discutidos à luz da análise de alguns aspectos metodológicos dos estudos das demandas judiciais por medicamentos, a saber: variabilidade das fontes de informação, completude dos dados e seleção da unidade de análise. 
O projeto de pesquisa do estudo foi aprovado pelo Comitê de Ética em Pesquisa da Escola Nacional de Saúde Pública/Fiocruz (Protocolo n. 139/2011) e pelo Comitê de Ética em Pesquisa em Seres Humanos da SESA/PR (Protocolo n. 363/2011).

\section{Resultados e discussão}

O estudo retornou o total de 694 demandas judiciais por medicamentos contra o sistema público de saúde do Estado do Paraná, no ano de 2009. O cadastro continuava ativo em $39,5 \%$ dos demandantes e estava inativo em $47,8 \% ; 12,7 \%$ dos demandantes tinham falecido. Os principais motivos que conduziram à inativação do cadastro dos pacientes da demanda judicial foram: mudança para o fornecimento programado no Componente Especializado da Assistência Farmacêutica (CEAF) (29,5\%); suspensão da liminar concedida pela justiça $(23,2 \%)$; término do tratamento (19,3\%); e suspensão por ordem médica (17,8\%). Vale ressaltar que ao menos dois motivos de inativação - suspensão da liminar e suspensão por ordem médica - sugerem a inadequação do tratamento demandado.

Em 2009, entre as 142 comarcas do Paraná ${ }^{17}, 74$ (52,1\%) originaram ações judiciais requerendo medicamentos, sendo as comarcas de Curitiba (20,5\%), Londrina (16,3\%), Região Metropolitana de Curitiba (8,9\%), Cascavel (6,2\%) e Maringá $(6,2 \%)$ as mais frequentes.

Os tipos de varas das comarcas distribuíram-se em Cível (46,7\%), Federal (42,7\%), Fazenda Pública (9,5\%), Família, Infância e Juventude (1\%) e Criminal $(0,1 \%)$, sendo que $6,6 \%$ das ações tramitaram nos Juizados Especiais. No que diz respeito ao tipo de ação impetrada, a demanda caracterizou-se por ação ordinária (38,9\%), civil pública $(25,1 \%)$, mandado de segurança $(22,8 \%)$, de obrigação de fazer $(9,5 \%)$, de conhecimento $(1,6 \%)$, cominatória $(1,2 \%)$ e outros tipos $(0,9 \%)$.

A justiça gratuita foi concedida a $85,5 \%$ dos demandantes. Em $37,2 \%$ das liminares concedidas estava prevista aplicação de medida constritiva ao seu descumprimento, e o mesmo ocorreu em $35,2 \%$ das sentenças proferidas. Houve apelação da sentença ao Tribunal do Segundo Grau em 387 casos, sendo que os apelantes foram os réus (38\%) - quando as instâncias gestoras responderam solidariamente -, o Estado do Paraná $(29,2 \%)$, a União (2,3\%), o demandante autor da ação original $(20,7 \%)$ e as partes $(9,8 \%)$. Quanto ao número de réus em cada ação, houve um único réu em $46,7 \%$ das ações, dois em $34,7 \%$ e três em $18,6 \%$.

No campo da saúde, identificaram-se duas variáveis de natureza político-administrativa importantes relacionadas com a gestão da assistência

\footnotetext{
${ }^{17}$ PARANÁ. Lei n. 14.277, de 30 de dezembro de 2003. Dispõe sobre a Organização e Divisão Judiciárias do Estado do Paraná e adota outras providências. Diário Oficial do Estado, 30/12/2003. Anexo 5826/788. Disponivel em: <http://www.legislacao.pr.gov.br/legislacao/listarAtosAno.do?action=exibir\&codAto=5826 \&codltemAto=49605>. Acesso em: 05 dez. 2011.
} 
farmacêutica. A primeira revela a distribuição geopolítica das ações judiciais segundo a Regional de Saúde (RS) de origem e sua cidade-sede, sendo que as cinco mais frequentes foram a $2^{\text {a }}$ RS-Curitiba (28,4\%), a $17^{\text {a }}$ RS-Londrina (16,9\%), a 10 ${ }^{a}$ RS-Cascavel (8,9\%), a $15^{\text {a }}$ RS-Maringá $(7,3 \%)$ e a $3^{\text {a }}$ RS-Ponta Grossa (4,9\%). A segunda variável pode ser considerada um indicador de qualidade da organização e execução do atendimento às ordens judiciais. Trata-se da presença da "Ficha Técnica" em 97,7\% das ações, como documento norteador da gestão da demanda judicial na SESA/PR.

Das características sociodemográficas, só foi possível determinar o indicador 4 (Tabela 1), que corresponde ao município de domicílio do autor, sendo o principal o Município de Curitiba (20,7\%). Os demais indicadores, que se baseavam nas variáveis "sexo", "idade", "renda anual per capita" e "ocupação", não puderam ser identificados, pois não foi possível ter acesso ao processo judicial na íntegra - em particular à petição inicial (Artigo 282, inciso II, do Código de Processo Civil $-\mathrm{CPC}^{18}$ ), peça na qual tais informações são facilmente encontradas. Pessoa ${ }^{19}$, Vieira e Zucchi ${ }^{20}$, Boing $^{21}$ e Machado ${ }^{22}$ identificaram em seus estudos características sociodemográficas utilizando a cópia do processo judicial como unidade de análise. Ainda assim,

Tabela 1. Características sociodemográficas do autor da ação judicial (Dimensão 1).

\begin{tabular}{lcc}
\hline $\begin{array}{l}\text { Indicador } 4 \text { - Proporção da população por município de domicílio do } \\
\text { autor da ação - abrangência de } 122 \text { municípios do total de } 399 \text { do } \\
\text { estado (n=694) }\end{array}$ & Frequência & $\%$ \\
\hline Curitiba & 144 & 20,7 \\
Londrina & 111 & 16,0 \\
Cascavel & 38 & 5,5 \\
Ponta Grossa & 28 & 4,0 \\
Maringá & 27 & 3,9 \\
Foz do lguaçu & 22 & 3,2 \\
Outros & 324 & 46,7 \\
\hline
\end{tabular}

Fonte: Cemepar (2009); elaboração própria.

\footnotetext{
${ }^{18}$ BRASIL. Lei Federal n. 5.869, de 11 de janeiro de 1973, que institui o Código de Processo Civil. Diário Oficial da União, Brasília, 17/01/1973.

${ }^{19}$ PESSOA, Nívia Tavares. Perfil das solicitações administrativas e judiciais de medicamentos impetradas contra a Secretaria de Saúde do Estado do Ceará. 2007. Dissertação (Mestrado) - Faculdade de Farmácia, Odontologia e Enfermagem. Universidade Federal do Ceará. Fortaleza, 2007.

${ }^{20}$ VIEIRA, Fabíola Sulpino; ZUCCHI, Paola. Distorções causadas pelas ações judiciais à política de medicamentos no Brasil. Revista de Saúde Pública, São Paulo, v. 41, n. 2, p. 214-222, abr. 2007.

${ }^{21}$ BOING, Alexandra Crispim. Política e Constituição: a judicialização do acesso a medicamentos em Santa Catarina. 2008. Dissertação (Mestrado) - Centro de Ciências Jurídicas, Políticas e Sociais. Universidade do Vale do Itajaí. Itajaí, 2008.

${ }^{22}$ MACHADO, Marina Amaral de Ávila et al. op. cit.
} 
a falta de informação dessas variáveis foi de $28 \%$ em até cerca de $90 \%$ nos processos estudados, exceto a variável "sexo", que foi identificada na totalidade dos processos.

Foi possível identificar e quantificar todas as variáveis que compõem a dimensão das características processuais das ações judiciais (Tabela 2). No entanto,

Tabela 2. Características processuais das ações judiciais (Dimensão 2).

\begin{tabular}{|c|c|c|}
\hline $\begin{array}{l}\text { Indicador } 1 \text { - Proporção das ações judiciais por representação do } \\
\text { autor da ação }(n=694)\end{array}$ & Frequência & $\%$ \\
\hline Advogado privado remunerado & 390 & 56,2 \\
\hline Promotor & 248 & 35,7 \\
\hline Defensor público & 49 & 7,1 \\
\hline Advogado privado dativo & 7 & 1,0 \\
\hline $\begin{array}{l}\text { Indicador } 2 \text { - Tempo mediano de decisão liminar ou antecipação de } \\
\text { tutela na primeira instância }(n=340)\end{array}$ & \multicolumn{2}{|c|}{$\begin{array}{l}\text { Mediana (dias) } \\
\quad 27\end{array}$} \\
\hline Indicador 3 - Tempo mediano da intimação da instância da saúde (n=338) & \multicolumn{2}{|c|}{$\begin{array}{l}\text { Mediana (dias) } \\
0\end{array}$} \\
\hline \multirow[t]{2}{*}{ Indicador 4 - Tempo mediano de entrega do medicamento $(n=570)$} & \multicolumn{2}{|c|}{$\begin{array}{l}\text { Mediana (dias) } \\
18\end{array}$} \\
\hline & Frequência & $\%$ \\
\hline $\begin{array}{l}\text { Indicador } 5 \text { - Proporção de concessão da liminar ou antecipação de } \\
\text { tutela ( } n=694)\end{array}$ & 658 & 94,8 \\
\hline $\begin{array}{l}\text { Indicador } 6 \text { - Proporção de ações judiciais com exigência judicial para } \\
\text { a concessão da liminar ou antecipação de tutela }(n=694)\end{array}$ & 487 & 70,2 \\
\hline $\begin{array}{l}\text { Indicador } 7 \text { - Proporção de sentenças favoráveis ao autor } \\
\text { ( } n=407 \text { sentenças proferidas) }\end{array}$ & 330 & 81,1 \\
\hline $\begin{array}{l}\text { Indicador } 8 \text { - Proporção de acórdãos favoráveis ao autor } \\
\text { ( } \mathrm{n}=237 \text { acórdãos registrados) }\end{array}$ & 219 & 92,4 \\
\hline $\begin{array}{l}\text { Indicador } 9 \text { - Razão de demandas extrajudiciais: } n=694 \text { demandas } \\
\text { judiciais / n=174 demandas extrajudiciais }\end{array}$ & \multicolumn{2}{|c|}{0,25} \\
\hline $\begin{array}{l}\text { Indicador } 10 \text { - Razão das ações judiciais coletivas ( } n=694): n=106 \\
\text { ações coletivas / } n=588 \text { ações individuais }\end{array}$ & \multicolumn{2}{|c|}{0,18} \\
\hline $\begin{array}{l}\text { Indicador } 11 \text { - Proporção de ações judiciais impetradas por tipo de } \\
\text { réu da ação }(n=694)\end{array}$ & Frequência & $\%$ \\
\hline União & 296 & 42,7 \\
\hline Estado & 225 & 32,4 \\
\hline Representante do Executivo & 153 & 22,0 \\
\hline Município & 14 & 2,0 \\
\hline Outro & 6 & 0,9 \\
\hline
\end{tabular}

Fonte: Cemepar (2009); elaboração própria. 
ficaram deficitários os cálculos dos tempos medianos de decisão liminar ou antecipação de tutela na primeira instância (47\%), da intimação da instância da saúde $(47,2 \%)$ e de entrega do medicamento (11\%). Comparativamente, as proporções faltantes dos tempos medianos foram bem menores ${ }^{23,24} \mathrm{em}$ estudos que tiveram acesso à íntegra do processo.

O conjunto de variáveis dessa dimensão norteará a análise das características processuais das ações judiciais, como exigência judicial de antecipação de tutela (presença de provas e documentos exigidos para a concessão da liminar), tempo de entrega do medicamento (da data da intimação da instância da saúde até a data de entrega do medicamento) e processo administrativo (conjunto de procedimentos junto à instância do Poder Executivo para a realização de um pedido sem intervenção do Poder Judiciário - ato de juiz) ${ }^{25}$, entre outras.

As variáveis das dimensões das características médico-sanitárias (Tabela 3) e político-administrativas (Tabela 4) foram identificadas na sua totalidade. A única dificuldade relacionada a essas dimensões foi a falta de acesso à prescrição médica ou sua cópia e a outros documentos médicos apensados ao processo em $35,2 \%$ dos casos, o que reduziu a frequência absoluta das variáveis "prescrição pelo nome genérico dos medicamentos", "prescrição exclusivamente pelo nome genérico dos medicamentos", "origem da prescrição médica segundo o sistema de saúde" e "outros documentos médicos apensados ao processo". Os demais dados da prescrição estavam contidos nos autos do processo judicial.

As variáveis da dimensão das características médico-sanitárias são importantes para a caracterização do objeto da demanda judicial - o medicamento - em termos de grupo terapêutico, custo, eficácia, força de recomendação e segurança, relacionando o pleito com a situação clínica do demandante, por meio de seu diagnóstico principal, exames complementares, laudos e perícias. Por outro lado, dizem também respeito a aspectos relacionados à Política de Medicamentos, tais como a presença na Rename, prescrição pelo nome genérico e presença de alternativa terapêutica no SUS ${ }^{26}$.

A partir das variáveis da dimensão das características político-administrativas, podem-se predizer ao menos cinco aspectos fundamentais ao

\footnotetext{
${ }^{23}$ SANT'ANA, João Maurício Brambati et al. Caracterização de demandas judiciais de fornecimento de medicamentos "essenciais" no Estado do Rio de Janeiro, Brasil. Cadernos de Saúde Pública, São Paulo, v. 26, n. 3, p. 461-471, mar. 2010.

${ }^{24}$ PEPE, Vera Lúcia Edais et al. Caracterização de demandas judiciais de fornecimento de medicamentos "essenciais" no Estado do Rio de Janeiro, Brasil. Cadernos de Saúde Pública, v. 26, n. 3, p. 461-471, 2010. ${ }^{25}$ PESSOA, Nívia Tavares. op. cit.

${ }^{26}$ PEPE, V. L. E.; VENTURA, M. (Org.). op. cit.
} 
Tabela 3. Características médico-sanitárias das ações judiciais (Dimensão 3).

\begin{tabular}{|c|c|c|}
\hline $\begin{array}{l}\text { Indicador } 1 \text { - Proporção de medicamentos por subgrupos } \\
\text { terapêuticos / farmacológicos / substâncias químicas }(n=973)\end{array}$ & Frequência & $\%$ \\
\hline \multicolumn{3}{|l|}{ Subgrupos Terapêuticos (54 subgrupos) } \\
\hline L01 (Agentes antineoplásicos) & 228 & 23,4 \\
\hline R03 (Fármacos para doenças obstrutivas das vias respiratórias) & 129 & 13,3 \\
\hline L04 (Imunossupressores) & 114 & 11,7 \\
\hline J05 (Antivirais de uso sistêmico) & 64 & 6,6 \\
\hline Outros & 438 & 45,0 \\
\hline \multicolumn{3}{|l|}{ Subgrupos Farmacológicos (89 subgrupos) } \\
\hline L01X (Outros agentes antineoplásicos) & 211 & 21,7 \\
\hline L04A (Imunossupressores) & 114 & 11,7 \\
\hline $\begin{array}{l}\text { R03B (Outros fármacos inalatórios para doenças obstrutivas das } \\
\text { vias respiratórias) }\end{array}$ & 106 & 10,9 \\
\hline N05A (Antipsicóticos) & 42 & 4,3 \\
\hline L03A (Imunoestimulantes) & 41 & 4,2 \\
\hline Outros & 459 & 47,2 \\
\hline \multicolumn{3}{|l|}{ Substâncias Químicas (215 fármacos diferentes) } \\
\hline L01XC02 (rituximabe) & 126 & 12,9 \\
\hline R03BB04 (brometo de tiotrópio) & 103 & 10,6 \\
\hline L04AB04 (adalimumabe) & 42 & 4,3 \\
\hline J05AB04 (ribavirina) & 31 & 3,2 \\
\hline L01XC03 (trastuzumabe) & 30 & 3,1 \\
\hline L03AB11 (alfapeginterferona-2a) & 30 & 3,1 \\
\hline L04AB01 (etanercepte) & 28 & 2,9 \\
\hline L04AB02 (infliximabe) & 26 & 2,7 \\
\hline G04BE03 (sildenafila) & 18 & 1,8 \\
\hline J05AF10 (entecavir) & 16 & 1.6 \\
\hline A10AE04 (insulina glargina) & 15 & 1,5 \\
\hline N05AH04 (quetiapina) & 15 & 1,5 \\
\hline C02KX01 (bosentana) & 14 & 1,4 \\
\hline A10AB04 (insulina lispro) & 12 & 1,2 \\
\hline Outros & 467 & 48,0 \\
\hline
\end{tabular}

Continua 
Pereira J. G., Pepe V. L. E.

Continuação

\begin{tabular}{|c|c|c|}
\hline $\begin{array}{l}\text { Indicador } 2 \text { - Proporção de medicamentos prescritos pelo nome } \\
\text { genérico ( } n=450 \text { prescrições) }\end{array}$ & 210 & 48,4 \\
\hline $\begin{array}{l}\text { Indicador } 3 \text { - Proporção de prescrições que utilizam exclusivamente } \\
\text { o nome genérico ( } \mathrm{n}=450 \text { prescrições) }\end{array}$ & 213 & 47,3 \\
\hline $\begin{array}{l}\text { Indicador } 4 \text { - Proporção de medicamentos requeridos que figuram } \\
\text { nas listas de medicamentos essenciais vigentes }(n=973)\end{array}$ & 120 & 12,3 \\
\hline $\begin{array}{l}\text { Indicador } 5 \text { - Ações judiciais contendo documentos adicionais, que } \\
\text { não a prescrição de medicamentos }(n=450)\end{array}$ & 450 & 100 \\
\hline \multicolumn{3}{|c|}{ Indicador 7 - Proporção de diagnósticos principais, por categoria diagnóstica $(n=694)$} \\
\hline \multicolumn{3}{|l|}{ Diagnósticos principais } \\
\hline J44 (Outras doenças pulmonares obstrutivas crônicas) & 102 & 14,7 \\
\hline B18 (Hepatite viral crônica) & 58 & 8,4 \\
\hline C83 (Linfoma não-Hodgkin difuso) & 46 & 6,6 \\
\hline M45 (Espondilite ancilosante) & 45 & 6,5 \\
\hline C50 (Neoplasia maligna da mama) & 33 & 4,8 \\
\hline F31 (Transtorno afetivo bipolar) & 22 & 3,2 \\
\hline L40 (Psoríase) & 21 & 3,0 \\
\hline I27 (Hipertensão pulmonar primária) & 20 & 2,9 \\
\hline E10 (Diabetes mellitus insulino-dependente - Tipo I) & 16 & 2,3 \\
\hline Outros & 311 & 47,7 \\
\hline $\begin{array}{l}\text { Indicador } 8 \text { - Proporção de pacientes com cadastro na instância de } \\
\text { saúde anterior à demanda judicial ( } n=694) \text {. }\end{array}$ & 60 & 8,6 \\
\hline $\begin{array}{l}\text { Indicador } 9 \text { - Razão de gasto de medicamentos demandados }{ }^{\mathrm{b}} \text { : Demanda } \\
\text { judicial = R\$ 35.004.454,00; Programação = R\$ 19.672.949,39 }\end{array}$ & \multicolumn{2}{|c|}{ 1,78: 1} \\
\hline \multirow[t]{2}{*}{$\begin{array}{l}\text { Indicador } 10 \text { - Proporção de medicamentos demandados com } \\
\text { alternativa terapêutica no Sistema Único de Saúde (SUS) (n=973) }\end{array}$} & Frequência & $\%$ \\
\hline & 913 & 93,8 \\
\hline
\end{tabular}

direcionamento das políticas e dos programas de assistência farmacêutica no SUS. O primeiro diz respeito ao registro na Anvisa, que aponta a eficácia e a segurança do medicamento demandado. O segundo reflete a distribuição dos medicamentos pelos componentes do bloco de financiamento e o terceiro, as ações judiciais que possuem medicamentos prescritos para indicação não 
Tabela 4. Características político-administrativas das ações judiciais (Dimensão 4).

\begin{tabular}{|c|c|c|}
\hline & Frequência & $\%$ \\
\hline Indicador 1 - Proporção de medicamentos registrados na Anvisa (n=973) & 968 & 99,5 \\
\hline \multicolumn{3}{|l|}{$\begin{array}{l}\text { Indicador } 2 \text { - Proporção de medicamentos, por componente do bloco de } \\
\text { financiamento da assistência farmacêutica }(n=973)\end{array}$} \\
\hline \multicolumn{3}{|l|}{ Componente } \\
\hline Atenção Básica & 62 & 6,4 \\
\hline Estratégico & 12 & 1,2 \\
\hline Excepcional & 257 & 26,4 \\
\hline APAC - Onco & 237 & 24,4 \\
\hline APAC - Oftalmo & 5 & 0,5 \\
\hline APAC - Outros & 21 & 2,2 \\
\hline Programa Estadual & 35 & 3,6 \\
\hline Não classificado & 344 & 35,4 \\
\hline $\begin{array}{l}\text { Indicador } 3 \text { - Proporção de ações judiciais que possuem ao menos um } \\
\text { medicamento prescrito para indicação de uso off label }(n=694)\end{array}$ & 134 & 19,3 \\
\hline $\begin{array}{l}\text { Indicador } 4 \text { - Proporção de ações judiciais que demandam ao menos } \\
\text { um medicamento que esteja fora dos componentes do bloco de } \\
\text { financiamento da assistência farmacêutica }(n=694)\end{array}$ & 507 & 73,1 \\
\hline $\begin{array}{l}\text { Indicador } 5 \text { - Proporção de ações judiciais que demandam ao menos } \\
\text { um medicamento do componente excepcional (especializado) da } \\
\text { assistência farmacêutica }(n=694)\end{array}$ & 206 & 29,7 \\
\hline
\end{tabular}

Fonte: Cemepar (2009); elaboração própria.

registrada (off label). O quarto refere-se às prescrições que possuem ao menos um medicamento ausente desses componentes e o quinto, às que possuem ao menos um medicamento do componente excepcional (especializado) ${ }^{27}$. Ainda com relação ao segundo indicador dessa dimensão, optou-se por extrapolar os componentes do bloco de financiamento da assistência farmacêutica, com o objetivo de configurar os medicamentos que possuem financiamento público em outras modalidades e que representam proporções bastante significativas neste estudo, como é o caso dos empregados nos procedimentos de alta complexidade no SUS (por exemplo, os medicamentos antineoplásicos e oftalmológicos) e dos que possuem financiamento próprio no Estado do Paraná.

${ }^{27}$ PEPE, V. L. E.; VENTURA, M. (Org.). op. cit. 
Uma nuance metodológica que vale registrar diz respeito à definição da unidade de análise. Estudos como os de Pessoa ${ }^{28}$, Vieira e Zucchi ${ }^{29}$, Boing ${ }^{30}$, Bomfim $^{31}$ e Machado ${ }^{32}$ a definem como o processo ou ação judicial, porém essa escolha não dá suporte à coleta de dados quando se trata de ações coletivas, nas quais os demandantes são múltiplos. Nesses casos, torna-se necessário converter a unidade de análise para a demanda individual, a fim de que as características dos autores e da ação possam ser estudadas individualmente. Este estudo considerou a demanda judicial individual como unidade de análise, possibilitando a ampliação do número de variáveis, o que enriqueceu o descritivo do conjunto das demandas - particularmente no que se refere àquelas decorrentes das decisões interlocutórias das ações coletivas, que, no caso da demanda judicial por medicamentos, podem ser publicadas individualmente, gerando registros e atos administrativos em diferentes tempos no setor saúde.

\section{Considerações finais}

Este estudo foi realizado para verificar, por meio de metodologia padronizada, se é possível identificar, em uma base de dados de demandas judiciais de medicamentos no SUS, a presença de todas as variáveis necessárias ao cálculo de indicadores de análise e monitoramento das demandas judiciais de medicamentos, de forma a contribuir para a padronização de futuros estudos e para o próprio monitoramento da demanda por parte da instância gestora da saúde.

Os resultados encontrados mostraram que as fontes utilizadas no estudo não foram suficientes para identificar e quantificar todas as variáveis propostas em suas dimensões, especialmente as que compõem alguns dos indicadores das características sociodemográficas. A determinação de indicadores da dimensão das características médico-sanitárias, baseados na prescrição médica ou em documentos médicos apensos ao processo judicial, ficou incompleta, uma vez que não foi possível ter acesso a esses documentos em parte significativa da demanda. Por outro lado, houve completude das variáveis utilizadas para os cálculos dos indicadores das dimensões das características processuais das ações judiciais e das características político-administrativas.

As falhas determinadas na aplicação do modelo metodológico proposto não se relacionaram diretamente ao método, mas à deficiência na qualidade das informações encontradas nas fontes disponíveis.

\footnotetext{
${ }^{28}$ PESSOA, Nívia Tavares. op. cit.

${ }^{29}$ VIEIRA, Fabíola Sulpino; ZUCCHI, Paola. op. cit.

${ }^{30}$ BOING, Alexandra Crispim. op. cit.

${ }^{31}$ BONFIM, Regina Lúcia Dodds. Agenda única de saúde: a busca do acesso universal e a garantia do direito à saúde. Tese (Doutorado) - Instituto de Medicina Social, Universidade do Estado do Rio de Janeiro. Rio de Janeiro, 2008.

${ }^{32} \mathrm{MACHADO}$, Marina Amaral de Ávila et al. op. cit.
} 
Conclui-se que, para aplicação uniforme dos indicadores básicos de monitoramento das demandas judiciais de fornecimento de medicamentos como instrumento de gestão, será necessário ao administrador da saúde ter em mãos uma cópia de cada processo judicial referente a cada demanda, assim como obter, por ocasião do cadastro para fornecimento dos medicamentos, os dados sociodemográficos.

\section{Referências}

ADVOCACIA GERAL DA UNIÃO - AGU. Intervenção judicial na saúde pública: panorama no âmbito da Justiça Federal e apontamentos na seara das Justiças Estaduais, 2012. Disponível em: <http://portalsaude.saude.gov.br/portalsaude/arquivos/pdf/2013/Abr/26/ Panoramadajudicializacao2013.pdf $>$. Acesso em: 20 maio 2013.

ANDRADE, Eli Iola Gurgel et al. A judicialização da saúde e a política nacional de assistência farmacêutica no Brasil: gestão da clínica e medicalização da justiça. Revista Médica de Minas Gerais, Belo Horizonte, v. 18, supl. 4, p. 46-50, 2008.

ANJOS, Renata Sacco dos. Judicialização e equidade no tratamento da hepatite C: estudo de caso sobre o tratamento com interferona alfa em um serviço de referência do SUS em Porto Alegre, RS, 2009. Dissertação (Mestrado) - Faculdade de Medicina, Universidade Federal do Rio Grande do Sul. Porto Alegre, 2009.

ASSOCIAÇÃO DOSSERVENTUÁRIOS DA JUSTIÇA DOESTADO DO PARANÁ - ASSEJEPAR. Consulta Processual. Disponível em: <http://www.assejepar.com.br >. Acesso em: 20 ago. 2011.

BOING, Alexandra Crispim. Política e Constituição: a judicialização do acesso a medicamentos em Santa Catarina. 2008. Dissertação (Mestrado) - Centro de Ciências Jurídicas, Políticas e Sociais. Universidade do Vale do Itajaí. Itajaí, 2008.

BONFIM, Regina Lúcia Dodds. Agenda única de saúde: a busca do acesso universal e a garantia do direito à saúde. Tese (Doutorado) - Instituto de Medicina Social, Universidade do Estado do Rio de Janeiro. Rio de Janeiro, 2008.

CAMARGO, Iara Alves. Análise das demandas judiciais para o tratamento da artrite reumatoide no Estado de São Paulo. 2011. Dissertação (Mestrado) - Universidade de Sorocaba. Sorocaba, 2011.

CHIEFFI Ana Luiza; BARATA Rita de Cássia Barradas. Ações judiciais: estratégia da indústria farmacêutica para introdução de novos medicamentos. Revista de Saúde Pública, São Paulo, v. 44, n. 3, p. 421-429, 2010.

FERRAZ, Octávio Luiz Motta; VIEIRA, Fabíola Sulpino. Direito à saúde, recursos escassos e equidade: os riscos da interpretação judicial dominante. Dados, v. 52, n. 1, p. 223-251, mar. 2009.

FIGUEIREDO, Tatiana Aragão. Análise dos medicamentos fornecidos por mandado judicial na Comarca do Rio de Janeiro: a aplicação de evidências científicas no processo de tomada de decisão. 2010. Dissertação (Mestrado) - Escola Nacional de Saúde Pública Sérgio Arouca, Fundação Oswaldo Cruz. Rio de Janeiro, 2010. 
LEITE, Silvana Nair et al. Ações judiciais e demandas administrativas na garantia do direito de acesso a medicamentos em Florianópolis-SC. Revista de Direito Sanitário, São Paulo, v. 10, n. 2, p. 13-28, 2009.

MACHADO, Marina Amaral de Ávila et al. Judicialização do acesso a medicamentos no Estado de Minas Gerais, Brasil. Revista de Saúde Pública, São Paulo, v. 45, n. 3, p. 590-598, 2011.

PARANÁ. Tribunal de Contas do Estado. Contas do Governador: relatório e parecer prévio, Exercício 2009. Curitiba: Tribunal de Contas do Estado; Instituto Rui Barbosa. 2010. Disponível em: <http://www.tce.pr.gov.br/contasdogoverno2009>. Acesso em: 16 out. 2012.

PEPE, Vera Lúcia Edais et al. Caracterização de demandas judiciais de fornecimento de medicamentos "essenciais" no Estado do Rio de Janeiro, Brasil. Cadernos de Saúde Pública, São Paulo, v. 26, n. 3, p. 461-471, 2010.

VENTURA, M. (Org.). Manual indicadores de avaliação e monitoramento das demandas judiciais de medicamentos. Rio de Janeiro: Fundação Oswaldo Cruz, Escola Nacional de Saúde Pública Sergio Arouca, Fundação Oswaldo Cruz. Rio de Janeiro, 2011.

PESSOA, Nívia Tavares. Perfil das solicitações administrativas e judiciais de medicamentos impetradas contra a Secretaria de Saúde do Estado do Ceará. 2007. Dissertação (Mestrado) - Faculdade de Farmácia, Odontologia e Enfermagem. Universidade Federal do Ceará. Fortaleza, 2007.

SANT'ANA, João Maurício Brambati. Essencialidade e assistência farmacêutica: um estudo exploratório das demandas judiciais individuais para acesso a medicamentos no Estado do Rio de Janeiro. Dissertação (Mestrado). Escola Nacional de Saúde Pública Sergio Arouca, Fundaçã Oswaldo Cruz. Rio de Janeiro, 2009.

et al. Caracterização de demandas judiciais de fornecimento de medicamentos “essenciais” no Estado do Rio de Janeiro, Brasil. Cadernos de Saúde Pública, São Paulo, v. 26, n. 3, p. 461-471, mar. 2010.

TEIXEIRA, Mariana Faria. Criando alternativas ao processo de judicialização da saúde: o sistema de pedido administrativo, uma iniciativa pioneira do Estado e Município do Rio de Janeiro. 2011. Dissertação (Mestrado) - Escola Nacional de Saúde Pública Sérgio Arouca, Fundação Oswaldo Cruz. Rio de Janeiro, 2011.

VIEIRA, Fabíola Sulpino; ZUCCHI, Paola. Distorções causadas pelas ações judiciais à política de medicamentos no Brasil. Revista de Saúde Pública, São Paulo, v. 41, n. 2, p. 214-222, abr. 2007.

WORLD HEALTH ORGANIZATION. WHO Collaborating Centre for Drug Statistics Methodology. ATC/DDD Index 2013. Disponível em: <http://www.whocc.no/atc_ddd_ index/>. Acesso em: 20 maio 2013. 


\section{Agradecimentos}

À Secretaria da Saúde do Estado do Paraná e ao Centro de Medicamentos do Paraná. Ao Centro Universitário de Maringá. À Coordenação de Aperfeiçoamento de Pessoal de Nível Superior (Capes), pela bolsa de doutorado concedida ao primeiro autor deste artigo.

José Gilberto Pereira - Doutor em Saúde Pública pela Escola Nacional de Saúde Pública da Fundação Oswaldo Cruz; Mestre em Ciências da Saúde pela Universidade Estadual de Maringá. Rio de Janeiro/RJ, Brasil. E-mail: jose.gilberto.pereira@gmail.com.

Vera Lúcia Edais Pepe - Doutora em Medicina (Medicina Preventiva) pela Universidade de São Paulo; Mestrado em Saúde Coletiva pela Universidade do Estado do Rio de Janeiro. Pesquisadora do Departamento de Administração e Planejamento em Saúde da Escola Nacional de Saúde Pública Sergio Arouca, da Fundação Oswaldo Cruz. Rio de Janeiro/RJ, Brasil. 